\title{
Dephasing of Cooper pairs and subgap electron transport in superconducting hybrids
}

\author{
Andrew G. Semenov, ${ }^{1,2}$ Andrei D. Zaikin, ${ }^{1,2,3}$ and Leonid S. Kuzmin ${ }^{2,4}$ \\ ${ }^{1}$ I.E.Tamm Department of Theoretical Physics, P.N.Lebedev Physics Institute, 119991 Moscow, Russia \\ ${ }^{2}$ Laboratory of Cryogenic Nanoelectronics, Nizhny Novgorod State Technical University, 603950 Nizhny Novgorod, Russia \\ ${ }^{3}$ Institut für Nanotechnologie, Karlsruher Institut für Technologie (KIT), 76021 Karlsruhe, Germany \\ ${ }^{4}$ Chalmers University of Technology, Goetenburg, Sweden \\ (Received 18 April 2012; published 23 October 2012)
}

\begin{abstract}
We argue that electron-electron interactions fundamentally restrict the penetration length of Cooper pairs into a diffusive normal metal $(N)$ from a superconductor $(S)$. At low temperatures, this Cooper pair dephasing length $L_{\varphi}$ remains finite and does not diverge at $T \rightarrow 0$. We evaluate the subgap conductance of $N S$ hybrids in the presence of electron-electron interactions and demonstrate that this length $L_{\varphi}$ can be directly extracted from conductance measurements in such structures.
\end{abstract}

DOI: 10.1103/PhysRevB.86.144529

PACS number(s): 74.45.+c, 73.40.Gk, 73.23.Hk

It is well known that a normal metal $(N)$ attached to a superconductor $(S)$ also acquires superconducting properties. ${ }^{1,2}$ This superconducting proximity effect is directly related to the phenomenon of Andreev reflection: ${ }^{3}$ At the NS interface Cooper pairs are converted into subgap quasiparticles (electrons), which can diffuse deep into the normal metal keeping information about a macroscopic phase of the superconducting condensate. Such macroscopic quantum coherence of electrons in the normal metal gets destroyed by thermal fluctuations, provided the corresponding inverse electron diffusion time (Thouless energy) becomes smaller than temperature $T$. As a result, superconducting coherence extends into a normal metal at a typical length $L_{T} \sim \sqrt{D / T}$ (where $D$ is the electron diffusion coefficient), implying that the whole normal metal can demonstrate superconducting properties at sufficiently low $T$.

This proximity-induced superconductivity manifests itself in a number of well-known phenomena, such as Meissner and Josephson effects in normal-superconducting hybrids ${ }^{4,5}$ as well as dissipative transport of subgap electrons across $N S$ interfaces. ${ }^{6}$ Provided the NS interface transmission is low, its corresponding subgap (Andreev) conductance $G$ remains rather small, being proportional to the second order in the barrier transmission. On the other hand, $G$ can be strongly enhanced at low energies due to nontrivial interplay between disorder and quantum interference of electrons in the normal metal, ${ }^{7-11}$ which leads to the so-called zero-bias anomaly (ZBA) $G \propto 1 / \sqrt{V}$ and $G \propto 1 / \sqrt{T}$ in the limit of low voltages and temperatures.

In this paper we will demonstrate that in the lowtemperature limit both superconducting proximity effect and ZBA in Andreev conductance are limited by dephasing of Cooper pairs due to electron-electron interactions in the normal metal. Note that Coulomb effects in subgap electron transport across $N S$ interfaces have been studied in several works: ${ }^{11-14}$ however, the decoherence effect of Coulomb interaction has not yet been addressed in a proper and complete manner. Below we will argue that fluctuating electromagnetic field produced by fluctuating electrons in a disordered normal metal destroys macroscopic coherence of electrons penetrating from a superconductor at a typical length scale $L_{\varphi}$. The existence of this length scale imposes fundamental limitations on the proximity effect in $N S$ hybrids at low temperatures $T \lesssim D / L_{\varphi}^{2}$. In this temperature range, the penetration depth of superconducting correlations into the normal metal is not given by the thermal length $L_{T}$ but is limited by the dephasing length $L_{\varphi}$, which-in contrast to $L_{T}$ - does not grow at $T \rightarrow 0$. We will evaluate Andreev conductance $G$ for $N S$ structures in the presence of electron-electron interactions and demonstrate that in the low-temperature limit, $G$ essentially depends on $L_{\varphi}$. This dependence allows to directly measure the dephasing length $L_{\varphi}$ in transport experiments with $N S$ hybrids.

It is also interesting to point out that the dephasing length $L_{\varphi}$ derived here for $N S$ systems up to a numerical prefactor coincides with zero-temperature decoherence length obtained within totally different theoretical framework ${ }^{15-19}$ for a different physical quantity - the so-called weak localization (WL) correction to the normal metal conductance. This agreement demonstrates fundamental nature of low-temperature dephasing by electron-electron interactions, which universally occurs in different types of disordered conductors, including normal-superconducting hybrids. On the other hand, as will be explained further below, dephasing of Cooper pairs by electron-electron interactions is in several important aspects different from that for single electrons in a normal metal encountered, e.g., in the WL problem.

The model and formalism. Below we will analyze a hybrid $S N$ structure, which consists of a normal metallic wire of crosssection $a^{2}$ and length $L \gg a$ attached to bulk superconducting and normal electrodes, as shown in Fig. 1. The contact between the wire and the $S$ electrode is achieved via a small tunnel barrier with cross-section $\Gamma$ and resistance $R_{I}$ strongly exceeding the wire resistance $R_{I} \gg R=L /\left(\sigma a^{2}\right)$, where $\sigma=2 e^{2} v D$ is the wire Drude conductivity, $e$ is the electron charge and $v$ is the density of states per spin direction.

In order to proceed, we will employ the Keldysh version of the nonlinear $\sigma$-model ${ }^{20,21}$ adapted to $S N$ structures. ${ }^{13}$ The effective action for our system defined on the Keldysh contour with forward (F) and backward (B) parts consists of two terms $S=S_{w}+S_{\Gamma}$ describing, respectively, diffusive motion of electrons in the wire,

$$
S_{w}[\check{Q}, \mathbf{A}, \Phi]=\frac{i \pi v}{4} \operatorname{Tr}\left[D(\check{\partial} \check{Q})^{2}-4 \check{\Xi} \partial_{t} \check{Q}+4 i \check{\Phi} \check{Q}\right],
$$




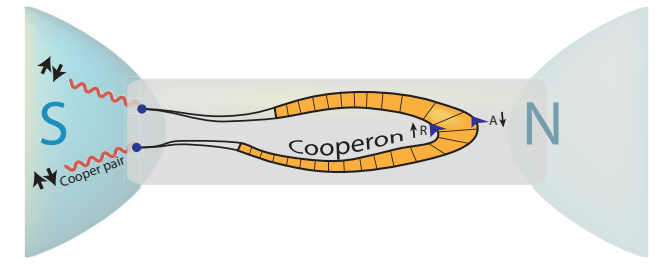

FIG. 1. (Color online) Hybrid $S N$ structure under consideration and the diagram describing conversion of a Cooper pair into a pair of electrons propagating inside the $N$ metal.

and tunneling between the wire and the superconductor,

$$
S_{\Gamma}[\check{Q}, \mathbf{A}, \Phi]=-\frac{i \pi}{4 e^{2} R_{I} \Gamma} \operatorname{Tr}_{\Gamma}\left[\check{Q}_{\mathrm{sc}}, \check{Q}\right],
$$

where $\check{Q}_{\text {sc }}$ and $\check{Q}$ are taken at superconducting and normal sides of the insulating barrier, $[x, y]$ denotes the commutator and "Tr" implies the trace over the matrix indices as well as the integration over times and coordinates. The covariant derivative is defined as

$$
\check{\partial} \check{Q}=\partial_{\mathbf{r}} \check{Q}-i[\check{\Xi} \check{\mathbf{A}}, \check{Q}], \quad \check{\Xi}=\left(\begin{array}{cc}
\hat{\sigma}_{z} & 0 \\
0 & \hat{\sigma}_{z}
\end{array}\right) .
$$

Here and below, $\hat{\sigma}_{x, y, z}$ denotes the set of Pauli matrices. Both parts of the action Eqs. (1) and (2) depend on the $4 \times 4$ dynamical matrix field $\mathscr{Q}$ satisfying the normalization condition $\breve{Q}^{2}=\breve{1} \delta\left(t-t^{\prime}\right)$ as well as on the fluctuating scalar and vector potentials $\Phi(\mathbf{r}, t)$ and $\mathbf{A}(\mathbf{r}, t)$, which are defined on the Keldysh contour and which account for the effect of electron-electron interactions. We define $\Phi^{ \pm}=\frac{1}{\sqrt{2}}\left(\Phi^{F} \pm \Phi^{B}\right)$ and $\mathbf{A}^{ \pm}=\frac{1}{\sqrt{2}}\left(\mathbf{A}^{F} \pm \mathbf{A}^{B}\right)$ and introduce the matrices

$$
\check{\Phi}=\left(\begin{array}{ll}
\Phi^{+} \hat{1} & \Phi^{-} \hat{1} \\
\Phi^{-} \hat{1} & \Phi^{+} \hat{1}
\end{array}\right), \quad \check{\mathbf{A}}=\left(\begin{array}{ll}
\mathbf{A}^{+} \hat{\mathbf{1}} & \mathbf{A}^{-} \hat{1} \\
\mathbf{A}^{-} \hat{1} & \mathbf{A}^{+} \hat{1}
\end{array}\right) .
$$

Perturbation theory and Gaussian integration. In what follows, we will restrict our consideration to energies well below the superconducting gap and set

$$
\check{Q}_{\mathrm{sc}}\left(t, t^{\prime}\right)=\left(\begin{array}{cc}
\hat{\sigma}_{y} & 0 \\
0 & \hat{\sigma}_{y}
\end{array}\right) \delta\left(t-t^{\prime}\right) .
$$

We will employ the so-called $\mathcal{K}$-gauge trick, ${ }^{20,21}$ which amounts to performing the gauge transformation $\check{Q}\left(\mathbf{r}, t, t^{\prime}\right) \rightarrow$ $e^{i \check{\Xi} \check{\mathcal{K}}(\mathbf{r}, t)} \check{Q}\left(\mathbf{r}, t, t^{\prime}\right) e^{-i \check{\Xi} \check{K}\left(\mathbf{r}, t^{\prime}\right)}$ in order to eliminate linear terms in both electromagnetic potentials and deviations from the $N$-metal saddle point

$$
\begin{gathered}
\check{Q}_{N}=\check{\mathcal{U}} \circ\left(\begin{array}{cc}
\hat{\sigma}_{z} & 0 \\
0 & -\hat{\sigma}_{z}
\end{array}\right) \check{\mathcal{U}}, \\
\check{\mathcal{U}}\left(t-t^{\prime}\right)=\left[\begin{array}{cc}
\delta\left(t-t^{\prime}-0\right) \hat{1} & -\frac{i T}{\sinh \left(\pi T\left(t-t^{\prime}\right)\right)} \hat{1} \\
0 & -\delta\left(t-t^{\prime}+0\right) \hat{1}
\end{array}\right] .
\end{gathered}
$$

This goal is accomplished with the choice of the $\mathcal{K}$ field obeying the following equations:

$$
\begin{gathered}
\Phi_{\mathcal{K}}^{+}(\mathbf{r}, t)=D \partial_{\mathbf{r}} \mathbf{A}_{\mathcal{K}}^{+}(\mathbf{r}, t) \\
-2 i D T \int d t^{\prime} \operatorname{coth}\left(\pi T\left(t-t^{\prime}\right)\right) \partial_{\mathbf{r}} \mathbf{A}_{\mathcal{K}}^{-}\left(\mathbf{r}, t^{\prime}\right), \\
\Phi_{\mathcal{K}}^{-}(\mathbf{r}, t)=-D \partial_{\mathbf{r}} \mathbf{A}_{\mathcal{K}}^{-}(\mathbf{r}, t),
\end{gathered}
$$

with $\Phi_{\mathcal{K}}(\mathbf{r}, t)=\Phi(\mathbf{r}, t)-\partial_{t} \mathcal{K}(\mathbf{r}, t)$ and $\mathbf{A}_{\mathcal{K}}(\mathbf{r}, t)=\mathbf{A}(\mathbf{r}, t)-$ $\partial_{\mathbf{r}} \mathcal{K}(\mathbf{r}, t)$. After this transformation, the action retains its initial form if one substitutes $\check{Q}_{\mathrm{sc}}\left(t, t^{\prime}\right) \rightarrow e^{-i \check{\Xi} \check{K}(\mathbf{r}, t)} \check{Q}_{\mathrm{sc}}\left(t, t^{\prime}\right) e^{i \check{\check{K}} \check{K}\left(\mathbf{r}, t^{\prime}\right)}$, $\Phi \rightarrow \Phi_{\mathcal{K}}$, and $\mathbf{A} \rightarrow \mathbf{A}_{\mathcal{K}}$.

Treating the tunneling term Eq. (2) perturbatively and performing the integration over the $\mathscr{Q}$ field, similar to Ref. 13, we arrive at the Andreev contribution to our action:

$$
S_{A}=-\frac{i}{32}\left(\frac{\pi}{e^{2} R_{I} \Gamma}\right)^{2}\left\langle\operatorname{Tr}_{\Gamma}\left[\check{Q}_{\mathrm{sc}}, \check{Q}\right] \operatorname{Tr}_{\Gamma}\left[\check{Q}_{\mathrm{sc}}, \check{Q}\right]\right\rangle_{Q} .
$$

The dependence of this term on the electromagnetic potentials is encoded both in $\check{Q}_{\text {sc }}$ and in the average of the $\check{Q}$ fields. Evaluating $S_{A}$ within the Gaussian approximation we will employ the parametrization ${ }^{20,21} \check{Q} \approx \check{Q}_{0}+i \check{Q}_{0} \circ \mathcal{U} \circ \check{W} \circ \mathcal{U}$ $-\frac{1}{2} \breve{Q}_{0} \circ \check{\mathcal{U}} \circ \check{W} \circ \check{W} \circ \check{\mathcal{U}}$ with

$$
\check{W}=\left(\begin{array}{cccc}
0 & c_{1}\left(\mathbf{r}, t, t^{\prime}\right) & d_{1}\left(\mathbf{r}, t, t^{\prime}\right) & 0 \\
\bar{c}_{1}\left(\mathbf{r}, t^{\prime}, t\right) & 0 & 0 & d_{2}\left(\mathbf{r}, t, t^{\prime}\right) \\
\bar{d}_{1}\left(\mathbf{r}, t^{\prime}, t\right) & 0 & 0 & c_{2}\left(\mathbf{r}, t, t^{\prime}\right) \\
0 & \bar{d}_{2}\left(\mathbf{r}, t^{\prime}, t\right) & \bar{c}_{2}\left(\mathbf{r}, t^{\prime}, t\right) & 0
\end{array}\right) .
$$

Here, $d_{i}$ and $c_{i}$ are, respectively, the diffuson and the Cooperon fields. Expanding the action $S_{w}$ up to the second order in these fields, one recovers four different contributions $S_{w}=$ $S_{w}^{(0,2)}+S_{w}^{(1,2)}+S_{w}^{(2,1)}+S_{w}^{(2,2)}$, where $S^{(i, j)}$ contains $i$ th power of the electromagnetic potentials and $j$ th power of $\check{W}$. By direct calculation, one can verify that the term $S^{(2,1)}$ depends only on the diffuson fields, which are irrelevant for the problem considered here. Hence, our action does not contain the first power of the Cooperon fields, and the corresponding propagator - the Cooperon $\mathcal{C}$ - can be obtained as a solution of a linear inhomogeneous equation containing the first and the second powers of electromagnetic potentials.

At this stage we would like to remark that the spin structure of the Cooperon analyzed here differs from that of the Cooperon encountered, e.g., in the WL problem in disordered normal metals. Indeed, representing the Cooperon as a sum of impurity ladder diagrams involving retarded $\left(G^{R}\right)$ and advanced $\left(G^{A}\right)$ Green functions one observes that the spin structure of the Cooperon responsible for the WL correction to the $N$-metal conductance is either $(\uparrow \uparrow)$ or $(\downarrow \downarrow)$, implying that both $G^{R}$ and $G^{A}$ correspond to either spin-up or spin-down states. In contrast, the spin structure of the Cooperon relevant for the proximity-induced superconductivity is either $(\uparrow \downarrow)$ or $(\downarrow \uparrow)$ simply because Cooper pairs are spin-singlets. It is straightforward to verify that only the Cooperon formed by antisymmetric combination $(\uparrow \downarrow-\downarrow \uparrow) / \sqrt{2}$ contributes to the subgap Andreev conductance of $N S$ structures. In the presence of electron-electron interactions, this Cooperon differs from that corresponding to other possible spin configurations already on the level of the first-order perturbation theory.

For illustration, let us consider the first-order diagrams depicted in Fig. 2. While in each of the cases $(\uparrow \uparrow),(\downarrow \downarrow)$, and $(\uparrow \downarrow+\downarrow \uparrow) / \sqrt{2}$, these diagrams cancel each other exactly at $T=0$, and in the limit of zero frequencies and momentum, no such cancellation occurs for the antisymmetric combination $(\uparrow \downarrow-\downarrow \uparrow) / \sqrt{2}$ because of extra minus sign encountered in this case. Thus, for the latter spin combination (which is 

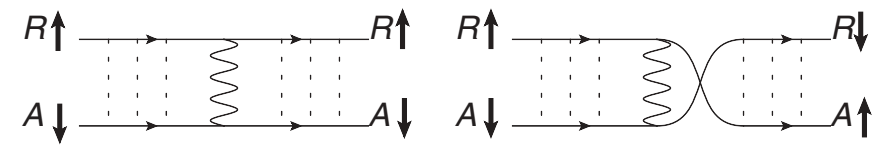

FIG. 2. First-order interaction corrections for the Cooperon. The dashed lines represent scattering on impurities and the wavy line accounts for electron-electron interactions.

only relevant here) nonvanishing zero-temperature dephasing is observed already within the first-order perturbation theory in the interaction.

Andreev conductance. Below we will proceed nonperturbatively and evaluate the subgap Andreev current $I$ to all orders in the interaction. Defining this current as

$$
I=\frac{e}{2} \int_{\Gamma} d^{2} \mathbf{r}\left\langle\delta S_{A} / \delta \mathcal{K}^{-}(\mathbf{r})\right\rangle_{\Phi}
$$

and calculating $S_{A}$ along the lines with the analysis, ${ }^{13}$ which now includes $\Phi_{\mathcal{K}}^{+}$and $\mathbf{A}_{\mathcal{K}}^{+}$, from Eq. (11), we obtain

$$
I=\frac{\pi T}{2 \nu e^{3}\left(R_{I} \Gamma\right)^{2}} \int_{\Gamma} d^{2} \mathbf{r} d^{2} \mathbf{r}^{\prime} \int d \tau \operatorname{Im} \frac{\left\langle\mathcal{P}\left(\mathbf{r}, \mathbf{r}^{\prime}, \tau ; t\right) e^{i e V \tau}\right\rangle_{\Phi}}{\sinh (\pi T \tau)},
$$

where $\mathcal{P}=\mathcal{C}\left(\mathbf{r}, \tau ; \mathbf{r}^{\prime}, 0 ; t-\tau / 2\right) e^{-2 i \mathcal{K}^{+}(\mathbf{r}, t)+2 i \mathcal{K}^{+}\left(\mathbf{r}^{\prime}, t-\tau / 2\right)}$ with the Cooperon $\mathcal{C}$ obeying the equation

$$
\left(2 \partial_{\tau}-i \Phi_{\mathcal{K}}^{+}(\mathbf{r}, T-\tau / 2)+i \Phi_{\mathcal{K}}^{+}(\mathbf{r}, T+\tau / 2)-D\left(\partial_{\mathbf{r}}+i \mathbf{A}_{\mathcal{K}}^{+}(\mathbf{r}, T-\tau / 2)+i \mathbf{A}_{\mathcal{K}}^{+}(\mathbf{r}, T+\tau / 2)\right)^{2}\right) \mathcal{C}\left(\mathbf{r}, \tau ; \mathbf{r}^{\prime}, \tau^{\prime} ; T\right)=\delta\left(\mathbf{r}-\mathbf{r}^{\prime}\right) \delta\left(\tau-\tau^{\prime}\right),
$$

and $V$ is an external voltage bias. Note that here and below we keep only the fields $\Phi^{+}$and $\mathcal{K}^{+}$neglecting $\Phi^{-}$and $\mathcal{K}^{-}$, which are irrelevant for dephasing of Cooper pairs. ${ }^{22}$

Resolving Eq. (13), we get

$$
\mathcal{P}\left(\mathbf{r}, \mathbf{r}^{\prime}, \tau ; t\right)=\frac{\theta(\tau) e^{i \mathcal{K}^{+}(\mathbf{r}, t-\tau)-i \mathcal{K}^{+}(\mathbf{r}, t)}}{2} \int_{\mathbf{x}(0)=\mathbf{r}^{\prime}}^{\mathbf{x}(\tau)=\mathbf{r}} \mathcal{D} \mathbf{x} e^{-\int_{0}^{\tau} d t^{\prime}\left(\frac{\left(\dot{x}\left(t^{\prime}\right)\right)^{2}}{2 D}-\frac{i}{2}\left\{\Phi^{+}\left[\mathbf{x}\left(t^{\prime}\right), t-\left(t^{\prime}+\tau\right) / 2\right]-\Phi^{+}\left[\mathbf{x}\left(t^{\prime}\right), t+\left(t^{\prime}-\tau\right) / 2\right]\right\}\right)}
$$

What remains is to perform a straightforward Gaussian average over $\Phi^{+}$fields as well as an average over diffusive trajectories.

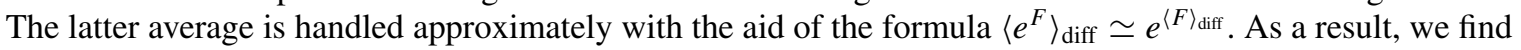

$$
\left\langle\mathcal{P}\left(\mathbf{r}, \mathbf{r}^{\prime}, \tau ; t\right)\right\rangle_{\Phi}=\mathcal{D}\left(\mathbf{r}, \mathbf{r}^{\prime} ; \tau\right) e^{-f\left(\mathbf{r}, \mathbf{r}^{\prime}, \tau\right)},
$$

with $f\left(\mathbf{r}, \mathbf{r}^{\prime}, \tau\right)=f_{t}(\mathbf{r}, \tau)+f_{b}\left(\mathbf{r}, \mathbf{r}^{\prime}, \tau\right)+f_{\mathrm{tb}}\left(\mathbf{r}, \mathbf{r}^{\prime}, \tau\right)$,

$$
\begin{gathered}
f_{t}(\mathbf{r}, \tau)=\frac{i}{2}\left[\mathcal{V}_{\mathcal{K} \mathcal{K}}^{++}(\mathbf{r}, \mathbf{r}, 0)-\mathcal{V}_{\mathcal{K} \mathcal{K}}^{++}(\mathbf{r}, \mathbf{r}, \tau)\right], \\
f_{b}\left(\mathbf{r}, \mathbf{r}^{\prime}, \tau\right)=i \int_{0}^{\tau} d t \int_{0}^{t} d t^{\prime} \int d^{d} \mathbf{x} d^{d} \mathbf{x}^{\prime}\left\{\mathcal{V}_{\Phi \Phi}^{++}\left[\mathbf{x}, \mathbf{x}^{\prime},\left(t-t^{\prime}\right) / 2\right]-\mathcal{V}_{\Phi \Phi}^{++}\left[\mathbf{x}, \mathbf{x}^{\prime},\left(t+t^{\prime}\right) / 2\right]\right\} \frac{\mathcal{D}(\mathbf{r}, \mathbf{x} ; \tau-t) \mathcal{D}\left(\mathbf{x}, \mathbf{x}^{\prime} ; t-t^{\prime}\right) \mathcal{D}\left(\mathbf{x}^{\prime}, \mathbf{r}^{\prime} ; t^{\prime}\right)}{\mathcal{D}\left(\mathbf{r}, \mathbf{r}^{\prime} ; \tau\right)},
\end{gathered}
$$

where $\mathcal{D}\left(\mathbf{x}, \mathbf{x}^{\prime}, t\right)$ is the diffusive propagator, $\mathcal{V}_{\Phi \Phi}^{++}\left(\mathbf{r}, \mathbf{r}^{\prime}, t-t^{\prime}\right)=$ $-2 i\left\langle\Phi^{+}(\mathbf{r}, t) \Phi^{+}\left(\mathbf{r}^{\prime}, t^{\prime}\right)\right\rangle_{\Phi}$, and $\mathcal{V}_{\mathcal{K} \Phi}^{++}, \mathcal{V}_{\mathcal{K} \mathcal{K}}^{++}$is defined analogously. The function $f_{\mathrm{tb}}$ is expressed via the correlator $\left\langle\mathcal{K}^{+} \Phi^{+}\right\rangle$. We chose to omit it here since $f_{\mathrm{tb}}$ remains much smaller than both $f_{t}$ and $f_{b}$.

Equations (12) and (15)-(17) define the central result of our work, which describes the effect of electronelectron interactions on the subgap current in diffusive $N S$ structures.

Quasi-1D structures. Below we will concentrate on quasi1D $N$-metal wires (Fig. 1) and set $\Gamma=a^{2}$. In this case the differential Andreev conductance $G(V)=d I / d V$ takes the form

$$
G=\frac{\pi T}{4 v e^{2} R_{I}^{2}} \int_{0}^{\infty} d \tau^{2} \frac{\mathcal{D}(0,0 ; \tau) \cos (e V \tau)}{\sinh (\pi T \tau)} e^{-f(0,0, \tau)}
$$

with $\mathcal{D}(0,0 ; \tau)=\vartheta_{2}\left(0, e^{-\tau / \tau_{D}}\right) /\left(2 L a^{2}\right)$, where $\vartheta_{2}$ is the second Jacobi theta function and $\tau_{D}=2 L^{2} /\left(\pi^{2} D\right)$ is the Thouless time. The function $f$ accounts for dephasing of Cooper pairs.
For $\pi T \tau \ll 1$, Eqs. (16) and (17) yield

$$
f(0,0, \tau) \simeq \frac{8}{g} \ln \left(\frac{\tau}{\tau_{R C}}\right)+\frac{\tau}{\tau_{\varphi}}+\sqrt{\frac{\pi \tau \tau_{c}}{4 \tau_{\varphi}^{2}}} \ln \left(\frac{\tau_{c}}{\tau}\right) .
$$

In the first term in Eq. (19) we defined dimensionless conductance $g=4 \pi \nu D a^{2} / L \gg 1$ and $\tau_{R C}=R C$, where $C$ is an effective capacitance. This term is caused by spatially uniform fluctuations of the scalar potential and matches with the results of Refs. 12 and 13. The remaining terms in Eq. (19) originate from nonuniform in space fluctuations in the $N$ metal and define the scales in our problem-Cooper pair decoherence time $\tau_{\varphi}=2 \pi v a^{2} \sqrt{2 D \tau_{c}}$ and decoherence length $L_{\varphi}=\sqrt{D \tau_{\varphi}}$, where $\tau_{c} \sim l / v_{F}$ sets a short time cutoff $^{15-17}$ and also $\tau_{\varphi} \gg \tau_{R C}$. Note that up to an unimportant prefactor of order one, $\tau_{\varphi}$ coincides with zero-temperature electron decoherence time evaluated, e.g., for the WL problem. ${ }^{15-17}$

At this point we emphasize that the agreement between the low-temperature dephasing length scales $L_{\varphi}$ found here for Cooper pairs and previously ${ }^{15-19}$ for single electrons is by no means a pure coincidence. Rather, this agreement 
reflects fundamental and universal nature of low-temperature quantum decoherence caused by electron-electron interactions in various types of disordered conductors. At the same time, the Cooperon encountered in the WL problem is in many respects different-both qualitatively and quantitatively - from that studied here. As we already indicated above, the most important difference is that the spin structure of our Cooperon (antisymmetric combination of spin-singlets) corresponds to that of a Cooper pair and is entirely different from that for the Cooperon in the WL problem. In addition, the Cooperon describing propagating Cooper pairs in the normal metal is naturally bound to the NS interface, which is obviously not the case in the WL problem. As a result, these two Cooperons are defined by formally different diagrammatic series and, hence, no a priori conclusions could possibly be drawn for our present problem from the Cooperon analysis developed for single electrons in disordered metals.

These differences have several important implications. For clarity, let us summarize the most important ones again: (i) Unlike single electrons in normal metals, Cooper pairs in NS structures get dephased already by uniform fluctuations of the scalar potential, as described by the first term in Eq. (19); (ii) unlike in the case of the WL problem, nonvanishing dephasing of Cooper pairs at $T=0$ occurs already within the first-order perturbation theory in the electron-electron interactions (see Fig. 2 and the corresponding discussion above); (iii) already at $T=0$ the Cooperon studied here decays differently as compared to the Cooperon in the WL problem, cf., e.g., our Eq. (19) and Eq. (28) in Ref. 17; and (iv) at not very low $T$, the temperature-dependent decay time for the Cooperon in NS systems is entirely different from that in the WL problem. $^{23}$

Turning to concrete results, we first consider the lowvoltage limit $\mathrm{eV} \ll T$. At high temperatures, $T \gg 1 / \tau_{\varphi}$, the penetration length of Cooper pairs into the $N$ metal is defined by $L_{T}$, while $L_{\varphi}$ is irrelevant and dephasing is only due to spatially uniform fluctuations described by the first term in Eq. (19). In this case, the results of Ref. 13 are reproduced and one finds $G(0) \propto T^{8 / g-1 / 2}$. At low temperatures, $T \ll 1 / \tau_{\varphi}$, on the contrary, $L_{T}$ becomes irrelevant and the penetration length of superconducting correlations into the $N$ metal is set by $L_{\varphi}$. Then, for the linear subgap conductance we obtain

$$
G(0) \simeq\left\{\begin{array}{cl}
\frac{1}{\sigma R_{I}^{2} a^{2}}\left(\frac{4 \tau_{R C}}{\tau_{D}}\right)^{8 / g} \frac{2 L \zeta\left(2-\frac{16}{g}\right)\left(2^{2-16 / g}-1\right)}{\pi^{2}}, & L \ll L_{\varphi}, \\
\frac{1}{\sigma R_{I}^{2} a^{2}} \frac{L_{\varphi}}{\sqrt{2 \pi}}\left(\frac{4 \tau_{R C}}{\tau_{\varphi}}\right)^{8 / g} \Gamma\left(\frac{1}{2}-\frac{8}{g}\right), & L \gg L_{\varphi},
\end{array}\right.
$$

where $\Gamma(x)$ is Euler gamma function and $\zeta(x)$ is Riemann zeta function. The dependence of $G(0)$ on $L$ at different temperatures is displayed in Fig. 3. At low $T$ it shows a pronounced maximum at $L \sim L_{\varphi}$, which can be conveniently used for experimental analysis of low-temperature dephasing of Cooper pairs in NS systems.

The same information can also be extracted from the nonlinear subgap conductance $G(V)$, which shows the ZBA peak at low voltages. ${ }^{7-11}$ At $T \rightarrow 0$ and $L \gtrsim L_{\varphi}$, the width of this peak is roughly determined by $\sim 1 / \tau_{\varphi}$.

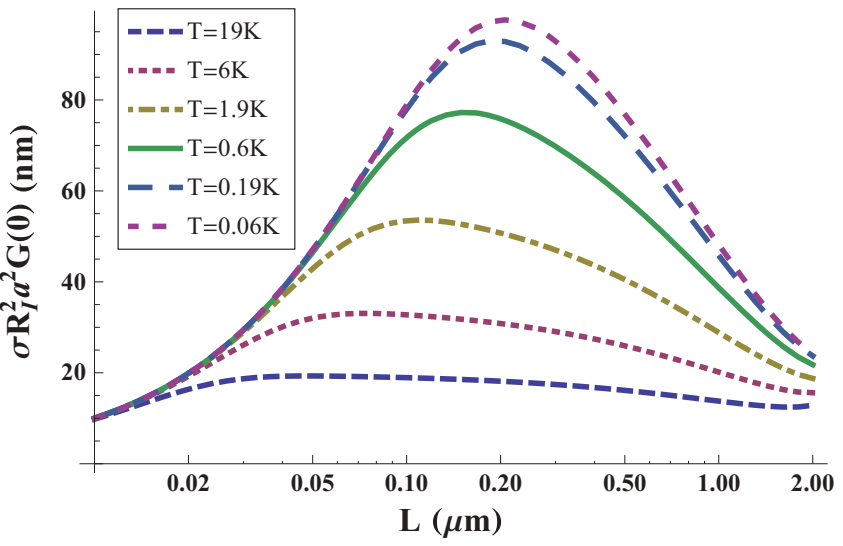

FIG. 3. (Color online) $G(0)$ as a function of $L$ for $a=10 \mathrm{~nm}$, $D=21 \mathrm{~cm}^{2} / \mathrm{s}$. For these parameter values, one finds $1 / \tau_{\varphi} \sim 0.6 \mathrm{~K}$ and $L_{\varphi} \sim 0.2 \mu \mathrm{m}$.

In particular, for $L \gg L_{\varphi}$ and $T=0$ we get

$$
G(V) \simeq \frac{1}{\sigma R_{I}^{2} a^{2}} \frac{L_{\varphi}}{\sqrt{2 \pi}}\left(\frac{4 \tau_{R C}}{\tau_{\varphi}}\right)^{8 / g} \operatorname{Re} \frac{\Gamma\left(\frac{1}{2}-\frac{8}{g}\right)}{\left(1+i e V \tau_{\varphi}\right)^{1 / 2-8 / g}} .
$$

The nonlinear subgap conductance $G(V)$ is depicted in Fig. 4 at different values of $L$.

Finally, we note that our analysis also allows us to determine the subgap conductance for other geometries. For example, in the $3 \mathrm{D}$ case the decoherence effect from spatially uniform fluctuations is negligible, ${ }^{13}$ and at $T \ll 1 / \tau_{\varphi}$ the dephasing of Cooper pairs in the $N$ metal is controlled by the second term in Eq. (19), with $\tau_{\varphi} \sim \sigma D^{1 / 2} \tau_{c}^{3 / 2} \propto D^{3}$.

In conclusion, we have demonstrated that electron-electron interactions yield dephasing of Cooper pairs penetrating from a superconductor into a diffusive normal metal. At low $T$ this phenomenon imposes fundamental limitations on the proximity effect in NS hybrids, restricting the penetration length of superconducting correlations into the $N$ metal to a temperature-independent value $L_{\varphi}$. This length scale can be probed by measuring the subgap conductance in NS systems.

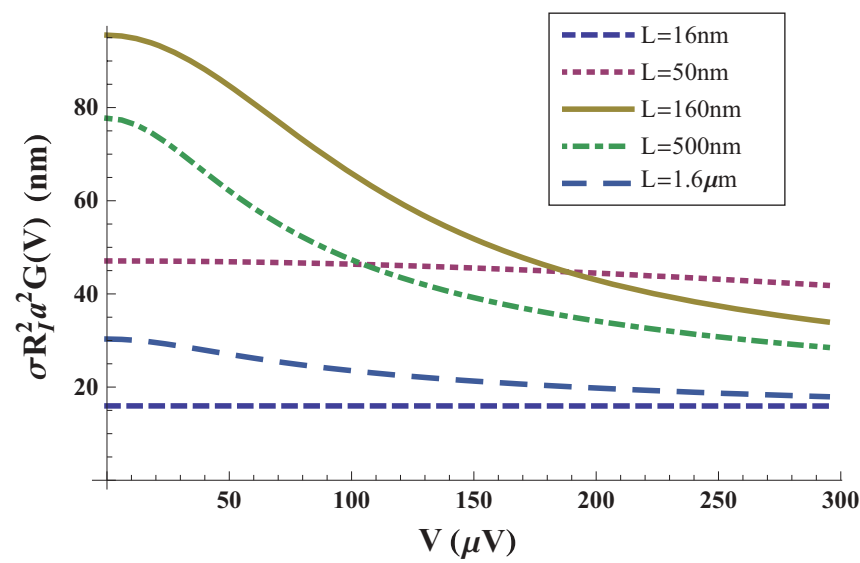

FIG. 4. (Color online) $G(V)$ at $T=0$ and different values of $L$. The parameters are the same as described in the caption of Fig. 3. 
We note that our results are qualitatively consistent with the experimental observations of Ref. 24, demonstrating that the low-temperature magnetoconductance of $N S$ structures is determined by phase-coherent electron paths with a typical size restricted by the temperature-independent dephasing length
$L_{\varphi}$ rather than by the thermal length $L_{T}$ diverging in the lowtemperature limit.

Acknowledgments. This work was supported by the Act 220 of the Russian Government (project 25) and by RFBR Grant No. 12-02-00520-a.
${ }^{1}$ P. G. de Gennes, Superconductivity of Metals and Alloys (Benjamin, New York, 1966).

${ }^{2} \mathrm{M}$. Tinkham, Introduction to Superconductivity (Dover, New York, 2004).

${ }^{3}$ A. F. Andreev, Sov. Phys. JETP 19, 1228 (1964).

${ }^{4}$ W. Belzig, F. K. Wilhelm, C. Bruder, G. Schön, and A. D. Zaikin, Superlattices Microstruct. 25, 1251 (1999).

${ }^{5}$ A. A. Golubov, M. Yu. Kupriyanov, and E. Il'ichev, Rev. Mod. Phys. 76, 411 (2004).

${ }^{6}$ G. E. Blonder, M. Tinkham, and T. M. Klapwijk, Phys. Rev. B 25, 4515 (1982).

${ }^{7}$ A. F. Volkov, A. V. Zaitsev, and T. M. Klapwijk, Physica C 210, 21 (1993).

${ }^{8}$ F. W. J. Hekking and Yu. V. Nazarov, Phys. Rev. Lett. 71, 1625 (1993).

${ }^{9}$ F. W. J. Hekking and Yu. V. Nazarov, Phys. Rev. B 49, 6847 (1994).

${ }^{10}$ C. W. J. Beenakker, B. Rejaei, and J. A. Melsen, Phys. Rev. Lett. 72, 2470 (1994).

${ }^{11}$ A. D. Zaikin, Physica B 203, 255 (1994).

${ }^{12}$ A. Huck, F. W. J. Hekking, and B. Kramer, Europhys. Lett. 41, 201 (1998).

${ }^{13}$ A. V. Galaktionov and A. D. Zaikin, Phys. Rev. B 73, 184522 (2006).
${ }^{14}$ A. V. Galaktionov and A. D. Zaikin, Phys. Rev. B 80, 174527 (2009).

${ }^{15}$ D. S. Golubev and A. D. Zaikin, Phys. Rev. Lett. 81, 1074 (1998).

${ }^{16}$ D. S. Golubev and A. D. Zaikin, Phys. Rev. B 59, 9195 (1999).

${ }^{17}$ D. S. Golubev and A. D. Zaikin, Phys. Rev. B 62, 14061 (2000).

${ }^{18}$ D. S. Golubev and A. D. Zaikin, New J. Phys. 10, 063027 (2008).

${ }^{19}$ D. S. Golubev and A. D. Zaikin, Physica E 40, 32 (2007).

${ }^{20}$ A. Kamenev and A. Andreev, Phys. Rev. B 60, 2218 (1999).

${ }^{21}$ A. Kamenev and A. Levchenko, Adv. Phys. 58, 197 (2009).

${ }^{22}$ This approximation is equivalent to dropping Coulomb blockade effects, which are negligible in the limit of large $g$ except at exponentially low voltages and temperatures. For instance, one can demonstrate ${ }^{13}$ that for $g \gg 1$ the terms $\left\langle\mathcal{K}^{+} \mathcal{K}^{-}\right\rangle$give negligible contribution to Andreev conductance as compared to that of the terms $\left\langle\mathcal{K}^{+} \mathcal{K}^{+}\right\rangle$

${ }^{23} \mathrm{We}$ do not present a detailed discussion of the Cooperon decay at nonzero $T$ here, since in this regime $L_{T}$ becomes shorter than $L_{\varphi}$ and, hence, the electron dephasing length becomes irrelevant for the problem under consideration.

${ }^{24}$ D. A. Dikin, M. J. Black, and V. Chandrasekhar, Phys. Rev. Lett. 87, 187003 (2001). In this experiment the electron decoherence length $L_{\varphi}$ was extracted from independent weak localization measurements and was found to be temperature independent in the regime $L_{\varphi} \lesssim L_{T}$. 